\title{
Caso 6/2020 - Jovem de 16 Anos, com Estenose Pulmonar Acentuada a Nível Valvar, após Correção de Tronco Arterial Comum pela Técnica de Barbero-Marcial no Primeiro Mês de Vida
}

\section{Case 6/2020 - 16-Year-Old Adolescent with Severe Pulmonary Stenosis At Valvar Level, After Correction of Truncus Arteriosus using the Barbero-Marcial Technique in the First Month of Life}

Edmar Atik ${ }^{\circledR}$ e Miguel Barbero-Marcial

Clínica privada do Dr Edmar Atik

\section{Dados Clínicos}

O recém-nascido, em insuficiência cardíaca com tronco arterial comum tipo I, foi corrigido com 15 dias de vida com $2.800 \mathrm{~g}$ de peso corporal pela técnica de Barbero-Marcial. Na ocasião fez-se a aproximação direta da via de saída do ventrículo direito (VD) com o tronco pulmonar e colocação de uma monocúspide em posição pulmonar.

A evolução foi adequada com o controle da insuficiência cardíaca e se manteve sem sintomas e com desenvolvimento físico normal. O exame clínico descartava lesões residuais como a insuficiência valvar pulmonar. No decorrer do tempo, assintomático, observou-se presença de sopro sistólico na área pulmonar, progressivo em intensidade ao lado de gradiente de pressão crescente na região da monocúspide pulmonar. Com 2 anos ele era de $25 \mathrm{mmHg}$, com 5 anos de $34 \mathrm{mmHg}$, com 7 anos de 40, com 13 anos de 90 e com 16 anos de 149 $\mathrm{mmHg}$. Não usa medicamentos específicos.

Exame físico: bom estado geral, eupneico, acianótico, pulsos normais. Peso: $60 \mathrm{Kg}$, Alt.: $165 \mathrm{~cm}, \mathrm{PA}: 110 / 70 \mathrm{mmHg}$, FC: $73 \mathrm{bpm}$. A aorta não era palpada na fúrcula.

No precórdio, ictus cordis não palpado e sem impulsões sistólicas na BEE. As bulhas cardíacas eram hiperfonéticas e auscultava-se sopro sistólico, $+/++/ 4$ de intensidade, rude, na área pulmonar e ao longo da BEE. Fígado não palpado e pulmões limpos.

\section{Exames Complementares}

Eletrocardiograma: Mostrava ritmo sinusal e sinais de bloqueio completo do ramo direito. $\mathrm{AQRS}=+160^{\circ}$, $\mathrm{AP}$ e $\mathrm{AT}=50^{\circ} \mathrm{C}$. A duração do QRS era de 0,13". Não havia potenciais de ventrículo esquerdo, com morfologia $r R^{\prime}$ em V1 e RS em V6.

\section{Palavras-chave}

Cardiopatias Congênitas; Insuficiência Cardíaca; Tronco Arterial/cirurgia; Técnica de Barbero-Marcial; Diagnóstico por Imagem.

Correspondência: Edmar Atik •

Consultório privado. Rua Dona Adma Jafet, 74, conj.73, Bela Vista. CEP 01308-050, São Paulo, SP - Brasil

E-mail: conatik@incor.usp.br

DOI: https://doi.org/10.36660/abc.20190490
Radiografia de tórax: Mostra área cardíaca moderadamente aumentada à custa dos arcos atrial e ventricular e trama vascular pulmonar normal. A cardiomegalia foi progressiva desde a correção cirúrgica, com índice cardiotorácico atual de 0,60 (Figura 1).

Ecocardiograma: Mostra o remendo interventricular bem posicionado e sem shunt residual. As cavidades direitas se mostram dilatadas em grau moderado e com disfunção ventricular. O VD está também hipertrofiado. Gradiente máximo entre VD e tronco pulmonar de 149 mmHg e médio de $86 \mathrm{mmHg}$. As dimensões eram: $\mathrm{Ao}=32, \mathrm{AE}=28, \mathrm{VD}=34$, $\mathrm{VE}=41$, septo $=$ parede posterior $=7$, função $\mathrm{VE}=66 \%$, $\mathrm{APD}=22$ e $\mathrm{APE}=26 \mathrm{~mm}$. Insuficiência pulmonar discreta.

Tomografia do coração: Mostrou os átrios de dimensões normais, VD com hipertrofia médio-apical e

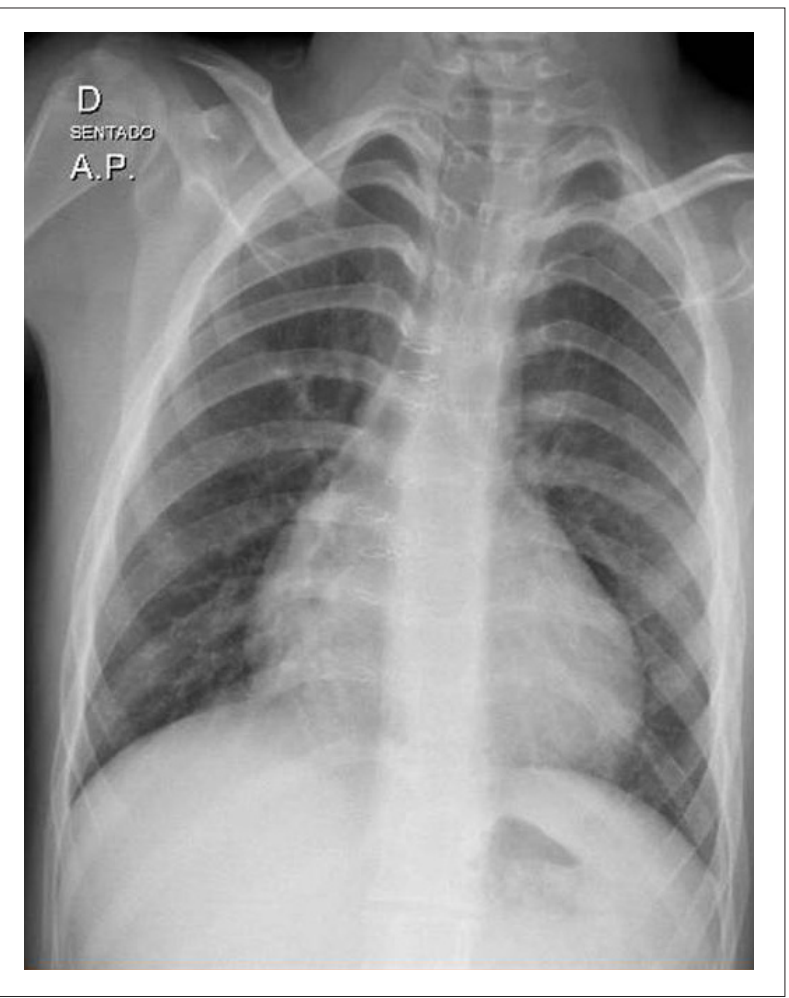

Figura 1 - Radiografia de tórax salienta o aumento moderado da área cardíaca à custa das cavidades direitas com trama vascular pulmonar normal. 


\section{Correlação Clínico-radiográfica}
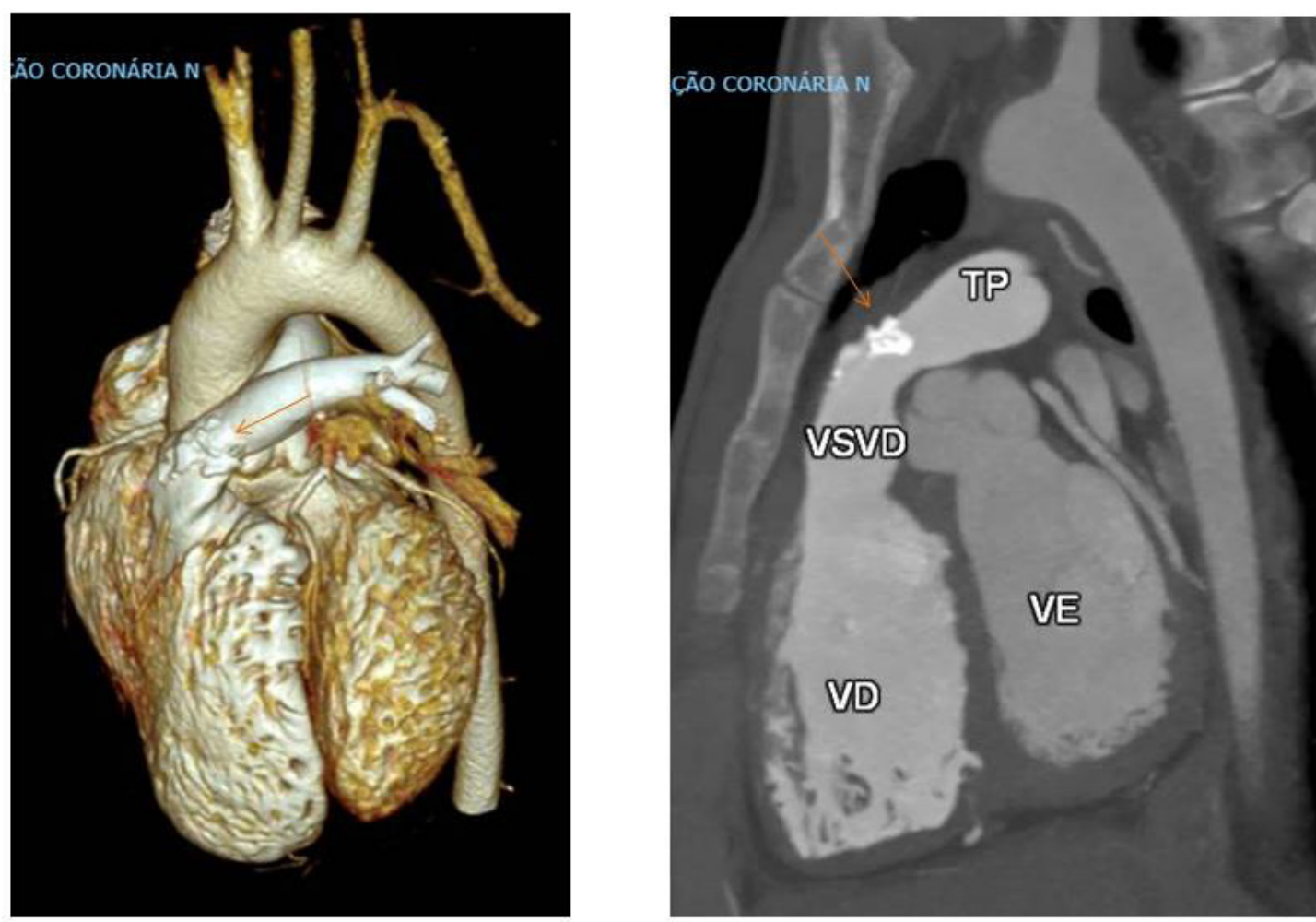

Figura 2 - Angiotomografia do coração em projeções de quatro cavidades e transversal salienta a hipertrofia miocárdica do VD e a via de saída do VD sem dilatação, mas com valva monocúspide nitidamente calcificada (setas). TP: tronco pulmonar; VD: ventrículo direito; VE: ventrículo esquerdo; VSVD: via de saída do ventrículo direito.

VDFVD $=135,2 \mathrm{ml} / \mathrm{m}^{2}$ e disfunção de $\mathrm{VD}=28 \%$. A via de saída do VD mostrava uma monocúspide calcificada e na planimetria da região a abertura valvar era de $0,95 \mathrm{~cm}^{2}$, com diâmetro de 14,3 x 6,2 cm. O septo interventricular estava íntegro e a aorta tinha calibre normal. Medidas de interesse: 1) Raiz de aorta: $35,4 \times 35,0 \mathrm{~mm}$ (Z-score 3,3). 2) Aorta ascendente: 27,6 × 25,2 mm. 3) Arco aórtico proximal: 22,1 x 20,4 mm - médio: 23,6 x 17,6 mm - distal: 21,0 x 17,6 mm 4) Aorta descendente: - proximal: $13,9 \times 13,5 \mathrm{~mm}$ - transição tóraco-abdominal: 11,4 x 9,6 mm. 5) Tronco pulmonar: 17,1 x 13,6 mm (Z-score -2,27). 6) Artéria pulmonar direita:16,3 x 13,0 mm (Z-score 0,35). 7) Artéria pulmonar esquerda: 11,7 x 10,1 mm (Z-score -0,96). 9) Ventrículo esquerdo: - Fração de ejeção: 49\% - Volume diastólico final indexado: $82,4 \mathrm{ml} / \mathrm{m}^{2}$.

Diagnóstico clínico: Tronco arterial comum tipo I operado precocemente sob a técnica de Barbero-Marcial com estenose pulmonar acentuada e progressiva observada na juventude, em paciente assintomático.

Raciocínio clínico: Os elementos clínicos evolutivos eram compatíveis com diagnóstico de estenose pulmonar que se instalou progressivamente desde a correção do defeito de base, o tronco arterial comum. A ausência de sintomas era esperada em presença da instalação insidiosa da obstrução através do tempo. A progressão maior da estenose ocorreu nos últimos três anos, provavelmente pela maior calcificação da monocúspide nesse período.
Diagnóstico diferencial: Lesão da valva pulmonar após correção cirúrgica pode ocorrer em toda situação na qual a valva pulmonar seja anteriormente reparada. Seu diagnóstico é simples através da presença de sopro sistólico na área pulmonar, acrescentado da hipertrofia miocárdica de VD nos exames de imagem.

Conduta: Em face da progressão do defeito residual a nível da valva pulmonar, já com caracteres adquiridos como hipertrofia miocárdica e disfunção de VD, a conduta de intervenção na região obstruída foi facilmente assimilada. Dada a anatomia adequada da região da valva pulmonar com diâmetro de $14 \mathrm{~mm}$ e sem dilatação da via de saída do VD, imaginou-se pertinente a abordagem da mesma por cateterismo cardíaco intervencionista. A colocação de prótese valvar tipo Melody foi a técnica de escolha, com o inconveniente da possibilidade de ocorrência de endocardite infecciosa em uma valva de origem de veia jugular bovina. As artérias coronárias bem afastadas da via de saída do VD favoreceram a suposição traçada.

Comentários: A técnica de Barbero-Marcial' para correção do tronco arterial comum, desenvolvida nos idos de 1989, é habitualmente acompanhada de insuficiência valvar pulmonar em face da dilatação da via de saída do VD na anastomose com o tronco pulmonar, tracionado em direção à mesma. Acompanha ainda a colocação de uma monocúspide, que analogamente ao que ocorre após a correção da tetralogia de 
Fallot, favorece também à evolução posterior de regurgitação pulmonar progressiva. Esses pacientes necessitam de correção do defeito residual e quase sempre por intervenção cirúrgica, em face da grande dilatação da região, que impossibilita a colocação de prótese endovenosa.

A preservação da via de saída mais estreita, como observada no caso apresentado, traz à baila a imaginação e discussão de como deveria se suceder em pacientes semelhantes operados, como mais comumente na tetralogia de Fallot. Tal fato poderia ocorrer mais vezes, desde que o cirurgião preservasse mais a via de saída do VD em uma área mais estreita, a permitir que evolutivamente a estenose pulmonar predominasse sobre a insuficiência valvar. Essa preferência decorre do fato de que a lesão miocárdica de sobrecarga de volume seja mais deletéria do que a estenose pulmonar, esta provocada mais pela calcificação da monocúspide.

O ideal é que esses pacientes sejam sempre devidamente monitorados, a fim de se preservar a condição preconizada para evolução mais favorável a mais longo prazo.

\section{Referências}

1. Barbero-Marcial M, Riso A, Atik E, Jatene A. A technique for correction of truncus arteriosus types I and II without extracardiac conduits. J Thorac Cardiovasc Surg. 1990;99(2):364-9. 